\title{
Reversible cerebral vasoconstriction syndrome with concurrent bilateral carotid artery dissection
}

\section{3(7) $491-495$}

(C) International Headache Society 2013

Reprints and permissions:

sagepub.co.uk/journalsPermissions.nav DOI: $10.1177 / 0333102413479836$ cep.sagepub.com

@SAGE

\author{
Anna Bayer-Karpinska', Maximilian Patzig², \\ Christopher Adamczyk ${ }^{3}$, Konstantinos Dimitriadis ${ }^{2}$, \\ Frank A Wollenweber', Martin Dichgans', Klaus Jahn ${ }^{3}$ and \\ Christian Opherk ${ }^{1,3}$
}

\begin{abstract}
Background: The pathophysiological basis of reversible cerebral vasoconstriction syndrome is poorly understood but carotid artery dissection has been discussed as a rare possible cause. So far, only single cases of unilateral carotid artery dissection and reversible cerebral vasoconstriction syndrome have been reported.

Case: Here, we describe the case of a 54-year old patient presenting to the emergency department with right hemiparesis, hypaesthesia and dysarthria. Furthermore, he reported two episodes of thunderclap headache after autosexual activity. Cerebral imaging showed ischaemic infarcts, slight cortical subarachnoid haemorrhage, bilateral carotid artery dissection and fluctuating intracranial vessel irregularities, compatible with reversible cerebral vasoconstriction syndrome. An extensive diagnostic work-up was normal. No typical trigger factors of reversible cerebral vasoconstriction syndrome could be found. The patient received intravenous heparin and the calcium channel blocker nimodipine. Followup imaging revealed no vessel irregularities, the left internal carotid artery was still occluded.

Conclusion: This case supports the assumption that carotid artery dissection should be considered as a potential trigger of reversible cerebral vasoconstriction syndrome, possibly by altering sympathetic vascular tone.
\end{abstract}

\section{Keywords}

Carotid artery dissection, reversible cerebral vasoconstriction syndrome, cerebral infarction, subarachnoid haemorrhage, thunderclap headache

Date received: 5 October 2012; revised: 17 December 20I2; accepted: 14 January 2013

\section{Introduction}

Reversible cerebral vasoconstriction syndrome (RCVS) is characterized by segmental vasoconstriction and vasodilatation of cerebral arteries resolving spontaneously within 3 months $(1,2)$. Its pathophysiological basis is poorly understood. Carotid artery dissection (CAD) has been discussed as a rare possible cause. So far, only single cases of unilateral CAD and RCVS have been reported, although all of them in combination with other more common causes, such as exposure to vasoactive substances or postpartum period (3-5). Herein, we describe a patient with spontaneous bilateral CAD and concurrent RCVS.

\section{Case report}

A 54-year-old man presented to the emergency department with acute right-sided weakness, numbness and slurred speech. Furthermore, he reported a dull holocephalic headache $5 / 10$ on the numeric rating scale (NRS) for 3 weeks and an upper respiratory tract infection lasting 2 weeks, which resolved under antibiotic treatment. Apart from smoking, no former diseases,

\footnotetext{
IInstitute for Stroke and Dementia Research, Ludwig-MaximiliansUniversity, Munich, Germany

${ }^{2}$ Department of Neuroradiology, Ludwig-Maximilians-University, Munich, Germany

${ }^{3}$ Department of Neurology, Ludwig-Maximilians-University, Munich, Germany

Corresponding author:

Anna Bayer-Karpinska, Institute for Stroke and Dementia Research, Ludwig-Maximilians-University, Max-Lebsche Platz 30, 8I 377 Munich, Germany.

Email: anna.bayer@med.Imu.de
} 


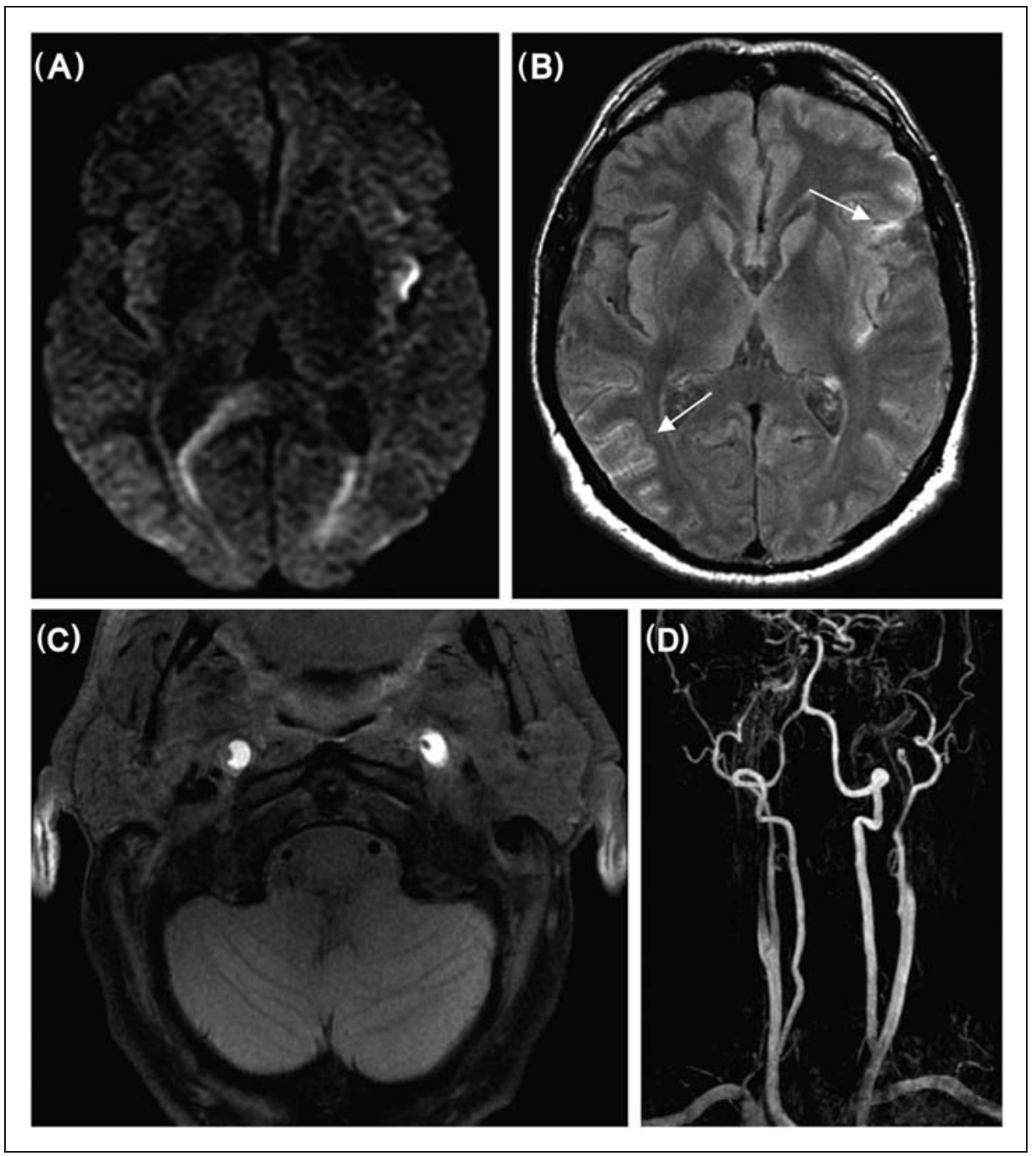

Figure I. Magnetic resonance imaging (MRI) findings in a patient with bilateral carotid artery dissection and reversible cerebral vasoconstriction syndrome. Cranial MRI showing ischaemic infarct (A, diffusion-weighted sequence) and cortical subarachnoid haemorrhage (B, FLAIR-sequence, arrows) on admission. Cervical MRI demonstrating bilateral carotid artery dissection with mural haematoma (C, TI-fat-saturated sequence) and occlusions of both internal carotid arteries (D, magnetic resonance angiography with contrast medium).

trauma, medication, alcohol or drug exposure were notable. Physical examination revealed right hemiparesis, hypaesthesia, dysarthria and elevated blood pressure of $160 / 80 \mathrm{mmHg}$. Initial magnetic resonance imaging (MRI) showed diffusion restrictions in the left posterior insula and adjacent white matter (Figure 1(A)) and FLAIR-hyperintensities within the left sylvian fissure and right parietal sulci (Figure 1(B)) compatible with cortical subarachnoid haemorrhage $(\mathrm{cSAH})$. In time of flight magnetic resonance angiography (TOFMRA), both internal carotid arteries (ICAs) were absent. cSAH was also detectable on non-contrast computed tomography (CT) and CT-angiography (CTA) confirmed proximal occlusion of both ICAs, suggesting bilateral CAD. Cerebral spinal fluid (CSF) showed 4288 erythrocytes/ $\mu 1$, increased protein level to $97 \mathrm{mg} /$ dl and 7 cells $/ \mu$, also compatible with cSAH. The patient was admitted to the stroke unit and received intravenous heparin (pTT 40-50 seconds) to avoid thrombus apposition. The next day's MRI including fat-saturated and black blood sequences confirmed bilateral CAD (Figure 1(C, D)) and, additionally, demonstrated irregularities in the left middle cerebral artery (MCA), right posterior cerebral artery (PCA) and right posterior communicating artery (Figure 2(A)). No contrast enhancement of the vessel walls was noted. In transcranial dopplersonography during the following 10 days a fluctuating increase in 


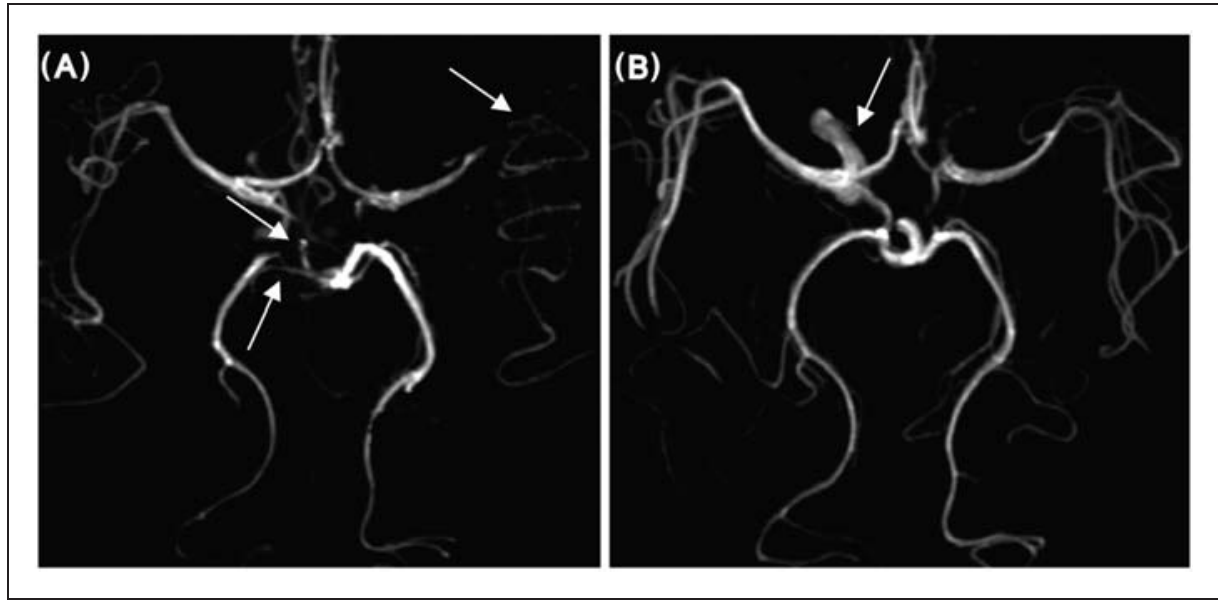

Figure 2. Time of flight magnetic resonance angiography (TOF-MRA) with irregularities (arrows) of the right posterior cerebral artery, right posterior communicating artery and left middle cerebral artery on admission (A) and at 3 months follow-up without intracranial vessel irregularities and with recanalised right internal carotid artery (B, arrow).

flow velocity in the left MCA was observed. Extensive blood and CSF analysis including parameters of systemic inflammatory conditions, various infectious agents and coagulopathies were normal. Dermatological and ophthalmological evaluations as well as repeated imaging of cervicocranial vessels were unsuggestive of fibromuscular dysplasia. A repeated interview revealed two episodes of sudden severe and throbbing headache reaching its maximum intensity of $8 / 10 \mathrm{NRS}$ within a few seconds and lasting about 30 minutes. These episodes of thunderclap headache occurred during autosexual activity 3 and 2 weeks before admission and were followed by a moderate headache, as previously described. With RCVS being the main differential diagnosis therapy was extended to the calcium channel blocker nimodipine. Eight days after admission MRA showed improvement of vessel irregularities and the right ICA was partly recanalised. The patient was discharged after nimodipine was tapered and oral anticoagulation was recommended for 6 months. At followup 3, 6 and 12 months later, no further attacks of thunderclap headaches or neurological symptoms had occurred. Intracranial MRA after 3 months revealed complete remission of intracranial vessel irregularities (Figure 2(B)). CTA and several dopplersonography studies during subsequent follow-up showed complete recanalisation of the right ICA with persistent occlusion of the left ICA. Vessel wall thickening, stenosis or dilations were absent and therefore no signs of fibromuscular dysplasia were found.

\section{Discussion}

Our patient suffered spontaneous bilateral CAD without any indication of trauma or inflammatory processes of the central nervous system. A preceding upper respiratory tract infection was the only environmental trigger factor we found. As proposed previously, infections can predispose individuals to CAD via endothelial damage or prothrombotic mechanisms (6). The patient was treated with antibiotics but did not use any nasal decongestants, which are known precipitating factors of RCVS. Recurrent thunderclap headaches and the results of intracranial imaging with complete resolution of vessel irregularities after 3 months strongly suggested the diagnosis of RCVS $(1,7)$. Digital subtraction angiography for definitive exclusion of other bleeding sources was resigned because of the high risk of harming the last supplying brain arteries (one of them a hypoplastic vertebral artery). Yet, none of the repeated CT- and contrast enhanced MR-scans revealed further pathologies. Furthermore, cSAH in this age group is most likely due to RCVS $(8,9)$.

A critical point in the clinical management of our case was the question of anticoagulation. In CAD with vessel occlusion or high-grade stenosis anticoagulation with heparin is often preferred because it is thought to more effectively prevent thrombus apposition and thromboembolic complications than aspirin (6). On the other hand, SAH is an obvious contraindication for anticoagulation in most situations. In our case, only focal cortical SAH was present and other bleeding sources, e.g. aneurysm, were absent on repeated imaging. Moreover, both ICAs were occluded, bearing a high thromboembolic risk. Therefore, we decided on moderate anticoagulation with heparin (pTT 40-50 seconds), which subsequently was changed to oral anticoagulation for 6 months.

In the absence of other typical causes of RCVS, such as exposure to vasoactive substances, at least two 
pathophysiological explanations can be discussed. First, a concomitance of CAD and RCVS as two different manifestations of a generalised vascular disorder, as previously suggested by Mellion et al. in a case of bilateral CAD and PRES (10). However, vertebral and basilar arteries were not affected, inflammatory processes were not noted and pathological changes disappeared spontaneously without anti-inflammatory therapy. Second, a pathophysiological relationship between CAD and RCVS could be hypothesised as previously discussed in the literature $(4,5,11)$. RCVS is thought to be related to changes in the cerebral vascular tone resulting in concurring vasoconstriction and vasodilatation, e.g. due to excessive sympathetic activity in combination with oxidative stress or endothelial dysfunction (11). Interestingly, Arnold et al. suggested that the distension of the carotid arterial wall due to mural haematoma could stimulate sympathetic fibres (12). Although we did not find clinical evidence for sympathetic activation in our patient, the lack of Horner's syndrome would support this hypothesis. An association between RCVS and unilateral CAD has been noted in single case reports (3-5). However, in all of them concomitant more common causes of RCVS were present. Herein, for the first time, we describe a case of spontaneous bilateral CAD and concurrent RCVS. Apart from sexual activity as a known trigger factor for thunderclap headaches in RCVS patients, no cause of RCVS could be found. Although a definite proof of a causal relationship between these two conditions cannot be established, in our opinion this case strengthens the notion that CAD should be considered as a potential cause of RCVS.

\section{Funding}

This research received no specific grant from any funding agency in the public, commercial or not-for-profit sectors

\section{Conflict of interest}

Dr Karpinska, Dr Patzig, Dr Adamczyk, Dr Dimitriadis and Dr Wollenweber report no disclosure. Professor Dichgans receives research support from the Vascular Dementia Research Foundation, Corona Foundation and Jackstaedt Foundation, receives research funding from Bayer Vital, Eisai, Essex, Ferrer and ICON, received travel expenses and honoraria for lectures from Bayer Vital, Boehringer Ingelheim Pharma, Heel, Bristol-Meyer Squibb, Lundbeck, Sanofi-Aventis and Shire as well as for educational activities not funded by industry, received honoraria for articles for Thieme, UpToDate and Kohlhammer, serves as assistant editor for Stroke and as an editorial board member of Cerebrovascular Diseases and serves as a consultant for Bayer Vital, Boehringer Ingelheim, Bristol-Meyer Squibb, and Heel. Professor Jahn receives research support from the German Research Foundation (DFG), the German Federal Ministry of Education and Research (BMBF) and Biogen Idec, received travel expenses and honoraria for lectures from Abbott, Boehringer-Ingelheim, Codman, GlaxoSmithKline, received honoraria for articles for Ecomed, Kohlhammer and Thieme. Dr Opherk receives research support from the DFG and the Dr Werner Jackstaedt Foundation, served on scientific advisory boards for Boehringer-Ingelheim and Bristol-Myers Squibb, received honoraria for lectures from Boehringer-Ingelheim, received honoraria for articles by Thieme and Kohlhammer.

\section{Clinical implications}

- First reported case of RCVS and concurrent spontaneous bilateral CAD.

- The absence of other known and well-described RCVS causes strengthens the notion that a pathophysiological relationship between bilateral CAD and RCVS might exist.

- Our observation is in line with the hypothesis that dysregulation of sympathetic tone might play a role in the development of RCVS.

\section{References}

1. Ducros A and Bousser MG. Reversible cerebral vasoconstriction syndrome. Pract Neurol 2009; 9: 256-267.

2. Calabrese LH, et al. Narrative review: Reversible cerebral vasoconstriction syndromes. Ann Intern Med 2007; 146: W34-W36.

3. Arnold M, et al. Postpartum cervicocephalic artery dissection. Stroke 2008; 39: 2377-2379.

4. Field DK, et al. Reversible cerebral vasoconstriction, internal carotid artery dissection and renal artery stenosis. Cephalalgia 2010; 30: 983-986.

5. Ducros A, et al. The clinical and radiological spectrum of reversible cerebral vasoconstriction syndrome.
A prospective series of 67 patients. Brain 2007; 130: 3091-3101.

6. Debette S and Leys D. Cervical-artery dissections: Predisposing factors, diagnosis, and outcome. Lancet Neurol 2009; 8: 668-678.

7. Schwedt TJ, Matharu MS and Dodick DW. Thunderclap headache. Lancet Neurol 2006; 5: 621-631.

8. Ducros A, et al. Hemorrhagic manifestations of reversible cerebral vasoconstriction syndrome: Frequency, features, and risk factors. Stroke 2010; 41: 2505-2511.

9. Werring DJ. Reversible cerebral vasoconstriction syndrome and intracranial hemorrhage: Some answers, many questions. Stroke 2010; 41: 2455-2456. 
10. Mellion ML and Rizvi S. Spontaneous bilateral carotid artery dissection and posterior reversible encephalopathy syndrome. Neurology 2005; 65: 1990.

11. Chen SP, Fuh JL and Wang SJ. Reversible cerebral vasoconstriction syndrome: Current and future perspectives. Expert Rev Neurother 2011; 11: 1265-1276.

12. Arnold M, et al. Pain as the only symptom of cervical artery dissection. J Neurol Neurosurg Psychiatry 2006; 77: 1021-1024. 